\title{
Les paralogues de SUMO et la réponse interféron
}

Ghizlane Maarifi, Laurent Dianoux, Sébastien Nisole,

Mounira K. Chelbi-Alix

Inserm UMR-S 1124, université Paris Descartes, 45, rue des Saints Pères, 75006 Paris, France. mounira.chelbi-alix@parisdescartes.fr

\section{Les interférons}

Les interférons (IFN) constituent une famille de cytokines impliquée dans diverses activités biologiques. Ils ont initialement été identifiés et nommés pour leur activité antivirale, mais ils exercent également des activités immuno-régulatrices, anti-prolifératives et apoptotiques. Les IFN activent la voie de signalisation JAK/STAT Uanus kinase/ signal transducer and activator of transcription) et induisent la transcription de plus de 300 gènes, les ISG (IFN-stimulated genes), dont les produits sont les médiateurs des activités biologiques des IFN [1].

Les IFN sont classés en trois types sur la base de leur antigénicité et de la spécificité de chaque type à un récepteur membranaire. L'IFN de type I regroupe sept interférons parmi lesquels deux sont majoritaires : les IFN $\alpha$ et $\beta$ [1]. La fixation des IFN de type I à leur récepteur permet l'activation de deux kinases, JAKl (Janus kinase 1) et Tyk2 (tyrosine kinase 2), ainsi que la phosphorylation des facteurs de transcription STATl et STAT2 (signal transducer and activator of transcription). Les hétérodimères phosphorylés STAT1-STAT2 s'associent à IRF9 (IFN regulatory factor 9) pour former le complexe ISGF3 (IFN stimulated gene factor 3) qui migre vers le noyau et se fixe sur des séquences promotrices ISRE (IFN stimulated response element) (Figure IA). Le facteur de transcription STATl est essentiel pour la formation du complexe ISGF3, comme le démontre l'observation que les souris invalidées pour le gène STATI ne répondent pas aux
IFN de type I [2]. II y a cependant de plus en plus de preuves que STAT2 peut former avec IRF9 un complexe alternatif «ISGF3-like », en l'absence de STATI, capable de générer une réponse aux IFN de type I et III [3]. Les cellules n'exprimant pas STATI (STATI ${ }^{-/-}$) ont un niveau d'expression de STAT2 très faible mais la réintroduction de STAT2 dans ces cellules restaure la réponse aux IFN de type I [4]. L'IFN de type II ne comprend qu'un seul membre, I'IFN $\gamma$. Après fixation de I'IFN $\gamma$ à son récepteur, STATl est phosphorylé [1] par JAK1 et JAK2 et forme des homodimères qui transloquent dans le noyau où ils induisent la transcription de gènes ayant une séquence GAS (gamma activation site) dans leur promoteur (Figure 1B).

L'IFN de type III, également appelé IFN $\lambda$, a été découvert plus récemment et comporte quatre membres, IFN $\lambda 1$ à IFN $\lambda 4$. Les IFN $\lambda$ se fixent à des récepteurs spécifiques exprimés à la surface des cellules épithéliales mais induisent la même signalisation que les IFN de type I.

\section{Les protéines SUMO}

Les protéines SUMO (small ubiquitin modifier) appartiennent à la famille des UBL (ubiquitin like proteins) [5]. Chez l'homme, il existe 4 paralogues ${ }^{1}$ de SUMO : SUMO1, SUMO2 et SUM03 qui sont ubiquitaires, contrairement à SUMO4 qui est spécifique des cellules rénales, de la rate et des ganglions lymphatiques. SUMO2 et SUMO3 possèdent des

${ }^{1}$ Produits de gènes homologues issus d'un même gène ancestral. séquences identiques à $95 \%$, mais qui ne sont identiques qu'à $50 \%$ avec la séquence de SuMOl [5]. Par conséquent, les protéines SUMO2 et SUMO3 sont reconnues par le même anticorps SUM02/3.

SUMO2 et SUM03, mais pas SUM01, possèdent le motif consensus $\Psi$ KXD/ $/ \varepsilon^{2}$ leur permettant de former des chaînes polySUM02/3. Néanmoins, SUMO1 peut terminer une chaîne poly SUM02/3.

La SUMOylation est une modification réversible qui implique la liaison covalente isopeptidique entre un résidu glycine dans la région C-terminale de SUMO et une lysine de la protéine substrat. La SUMOylation est impliquée dans de nombreux processus cellulaires tels que la régulation transcriptionnelle, la dégradation des protéines et la défense antivirale. Depuis la découverte de SUMO en 1996, le nombre de ses substrats identifiés dépasse les 500 [6].

\section{SUMO inhibe la phosphorylation} de STATl en réponse à I'IFN $\alpha$ et l'IFN $\gamma$ Un travail antérieur avait montré que STATI rendue non SUMOylable est hyperphosphorylée en réponse à I'IFN $\gamma$ [7]. Nous avons montré [8] que dans les cellules qui expriment en stable les différents paralogues de SUMO : (1) STATI interagit avec SUMO et est SUMOylée, ce qui réduit sa phosphorylation et sa translocation vers le noyau en réponse aux IFN de type I et type II ; (2) I'IFN

\footnotetext{
$2 \Psi$ représente un acide aminé hydrophobe, $K$ la lysine qui forme la liaison isopeptidique avec le SUMO, $x$ un acide aminé quelconque et $D / \varepsilon$ un acide aspartique ou un acide glutamique.
} 


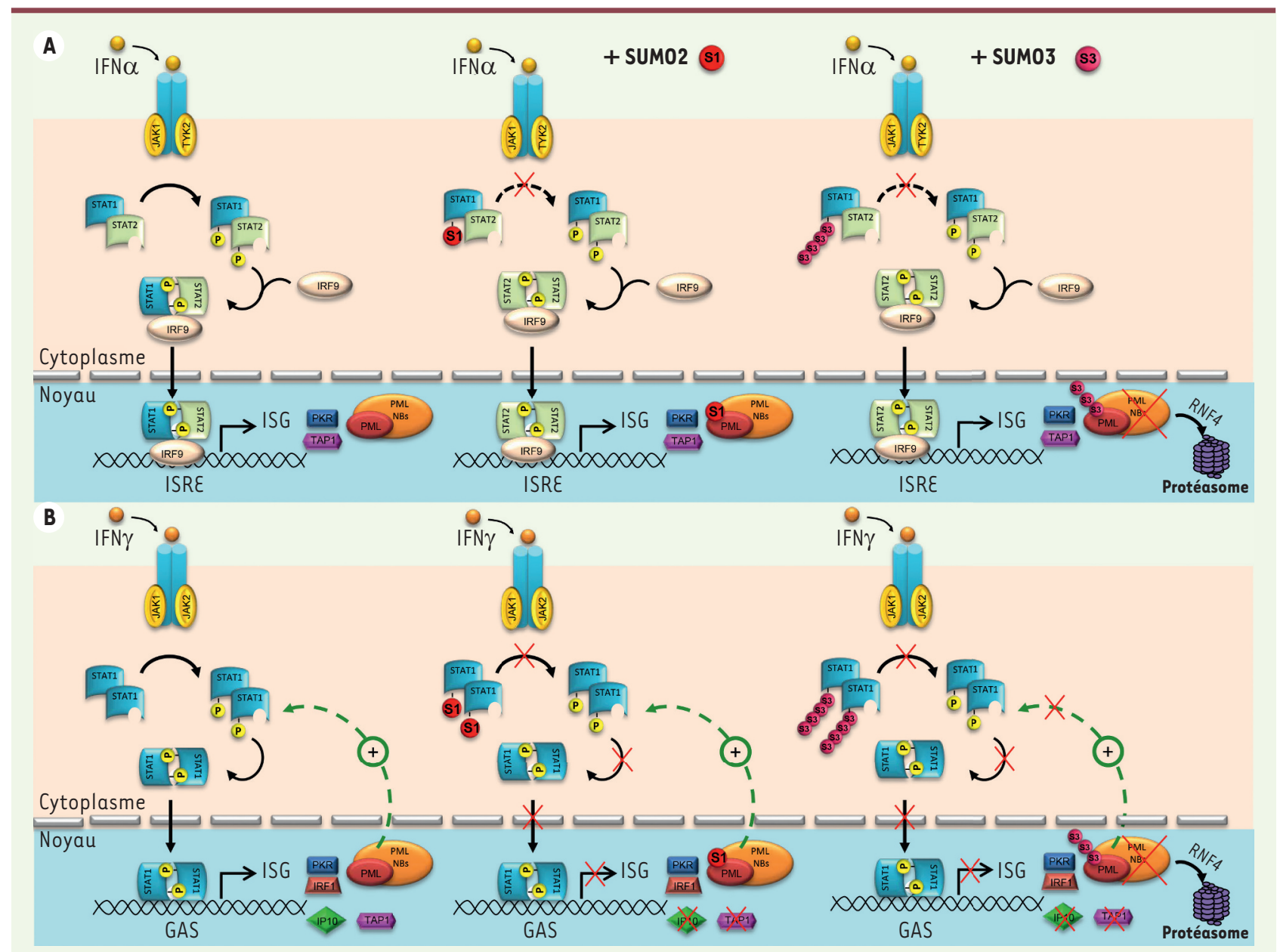

Figure 1. Modèle pour l'inhibition du signal de transduction de l'IFN par SUMO1 et SUM03. A. Fixation de l'IFN $\alpha$ à son récepteur induit la phosphorylation de STAT1 et STAT2 qui forment des hétérodimères STAT1-STAT2. Ces hétérodimères s'associent au facteur de transcription IRF9 pour former le complexe ISGF3 qui migre vers le noyau et se fixe au motif ISRE. II active alors la transcription des ISG tels que PML, TAPI et PKR. L'expression de SUMOI ou SUM03 inhibe la phosphorylation de STATl en réponse à I'IFN $\alpha$ mais n'altère pas la liaison de ISGF3 à l'ISRE. Le complexe alternatif «ISGF3-like » est en fait formé par l'homodimère STAT2-STAT2 et IRF9. L'IFN $\alpha$ induit la conjugaison de PML à SUM03 ce qui entraîne sa dégradation via le protéasome. Les réponses biologiques médiées par PML dans les cellules traitées à l'IFN $\alpha$ sont altérées. B. Fixation de l'IFN $\gamma$ à son récepteur induit la phosphorylation de STATl qui forme des homodimères. Ces homodimères migrent vers le noyau, se fixent au motif GAS et activent la transcription des ISG tels que PML, PKR, IRFl, TAPl et IP10. PML régule positivement le signal de transduction et la transcription en réponse à l'IFN $\gamma$ en induisant une plus forte activation de STATl. L'expression de SUMOI ou SUMO3 inhibe la phosphorylation de STATl et la transcription en réponse à I'IFN $\gamma$. L'IFN $\gamma$ induit la conjugaison de PML à SUM03 ce qui entraîne sa dégradation via le protéasome et une plus forte inhibition de la transcription en réponse à I'IFN $\gamma$. GAS : gamma activation site; IFN: interféron; IPI0 : IFN gamma-induced protein 10 ; IRFI/9 : IFN regulatory factor $1 / 9$; ISG : IFN-stimulated gene ; ISGF3 : IFN stimulated gene factor 3 ; ISRE : IFN stimulated response element ; JAK1/2 : Janus kinase $1 / 2$; PKR : doublestranded RNA-dependent protein kinase; PML : promyelocytic leukaemia ; RNF4 : RING finger protein 4 ; STAT : signal transducer and activator of transcription; SUMO : small ubiquitin modifier ; TAPI : transporter associated with antigen processing 1.

régule négativement son propre signal en augmentant la SUMOylation de STATl ; et (3) bien que SUMO diminue la phosphorylation de STATl en réponse à I'IFN $\alpha$, il n'affecte ni la phosphorylation de STAT2 ni sa translocation vers le noyau (Figure IA).
Comme attendu, la réduction de l'activation de STATI par SUMO en réponse à I'IFN $\gamma$ entraîne une diminution de la liaison de l'homodimère phosphorylé STATI-STATl à la séquence GAS (Figure 1B). Par contre, la diminution de la phosphorylation de STATl en réponse à I'IFN $\alpha$ n'altère pas la liaison du complexe ISGF3 à la séquence ISRE, le complexe alternatif «ISGF3-like » ne nécessitant pas STATl phosphorylé puisqu'il est formé par l'homodimère phosphorylé STAT2-STAT2 et IRF9 (Figure 1A) [8]. 


\section{SUMO inhibe la transcription} en réponse à I'IFN $\gamma$ sans altérer celle de I'IFN $\alpha$

L'effet de SUMO sur la transcription a été testé en utilisant les séquences promotrices spécifiques de l'IFN $\alpha$ et l'IFN $\gamma$, respectivement ISRE et GAS, fusionnées à la luciférase. L'expression de SUMOI ou de SUMO3 n'altère pas la transcription en réponse à l'IFN $\alpha$ mais inhibe la transcription en réponse à I'IFN $\gamma$ (Figure 1). Fait intéressant sur lequel on reviendra plus loin, l'inhibition de la transcription en réponse à IFN $\gamma$ est de $30 \%$ dans les cellules qui surexpriment SUMOl et de $80 \%$ dans les cellules qui surexpriment SUM03.

\section{L'augmentation de la conjugaison de PML à SUMO en réponse à I'IFN entraîne sa dégradation via le protéasome} PML (promyelocytic leukaemia) est l'une des protéines produite par les gènes dont la transcription est induite par les IFN, les ISG (IFN-stimulated genes). PML permet la formation de structures appelées corps nucléaires PML (PML NBs, PML nuclear bodies) (Figure 1) [9-12] $(\rightarrow)$.

II est connu que I'arsenic augmente la SUMOylation de PML et que SUM02/3 peut agir comme un signal de recrutement de RNF4 (RING finger protein 4), une SUMO- $\varepsilon 3$-ligase

\section{$(\rightarrow)$ Voir la Nouvelle de T. Regad et M.K. Chelbi-Alix, $\mathrm{m} / \mathrm{s} \mathrm{n}^{\circ} 1$, janvier 2002, page 25 , et la Synthèse de M.A. Maroui et al., $m / s n^{\circ} 8-9$, août-septembre 2014, page 765}

qui se lie aux polychaînes SUMO de PML (Figure 1). Ceci entraîne l'ubiquitination de PML et sa dégradation dans les cellules traitées à l'arsenic [13, 14]. Dans les cellules qui expriment SUMO3, le traitement à I'IFN $\alpha$ ou l'IFN $\gamma$ induit la conjugaison de PML à SUMO3 à des temps précoces. L'expression de l'ARNm de PML a donc lieu sans augmentation de la quantité de la protéine PML, celle-ci étant dégradée via le protéasome. Cette dégradation de PML n'est pas observée quand ces cellules sont traitées avec un inhibiteur du protéasome ou déplétées en RNF4.
L'inhibition massive de la réponse transcriptionnelle de I'IFN $\gamma$ par SUMO3 est due à la dégradation de PML

L'expression de chaque isoforme nucléaire de PML régule positivement la phosphorylation de STATl et la transcription en réponse à I'IFN $\gamma$ (Figure IB) [15]. La déplétion en RNF4 stabilise PML et augmente donc la phosphorylation de STATl et la transcription en réponse à I'IFN $\gamma[8]$.

Comme mentionné précédemment, la transcription en réponse à IFN $\gamma$ est inhibée de $30 \%$ par SUMOl et de $80 \%$ par SUM03 [8]. Cette plus forte inhibition est en fait due, dans les cellules exprimant SUM03, à la dégradation de PML SUMOylée par le protéasome. En effet, lorsque ces cellules sont déplétées en RNF4, on observe une restauration de la concentration en protéine $P M L$, ce qui ramène l'inhibition de la transcription de $80 \%$ à $30 \%$.

\section{Conclusions}

L'inhibition de la voie de signalisation de I'IFN $\gamma$ par SUMO est associée à une réduction de l'apoptose, de l'inhibition de la croissance cellulaire et du chimiotactisme. De façon réciproque, l'inhibition de la SUMOylation par diminution de l'expression d'Ubc9 (ubiquitin conjugating enzyme 9), unique enzyme de conjugaison, entraîne en réponse à l'IFN $\gamma$ une augmentation de la phosphorylation de STATl et des réponses biologiques. L'expression de SUMO rend donc les cellules résistantes à l'IFN $\gamma$. De plus, SUM03, mais pas SUM01, bloque la capacité de l'IFN $\alpha$ et de I'IFN $\gamma$ à augmenter l'expression de la protéine PML et réduit la capacité de I'IFN à conférer la résistance au virus de l'encéphalomyocardite (EMCV) [8]. Ceci est en accord avec des résultats précédents montrant que PML joue un rôle clé pour inhiber l'EMCV [16].

Pris dans leur ensemble, nos résultats mettent en évidence un rôle nouveau de SUMO dans la modulation de la réponse aux interférons de type I et II. $\diamond$ SUMO paralogs and interferon response

\section{REMERCIEMENTS}

Ce travail a été financé par l'Agence Nationale de la Recherche (ANR 11BSV3002803)

\section{LIENS D'INTÉRÊT}

Les auteurs déclarent n'avoir aucun lien d'intérêt concernant les données publiées dans cet article.

\section{RÉFÉRENCES}

1. Schneider WM, Chevillotte MD, Rice CM. Interferonstimulated genes: a complex web of host defenses. Annu Rev Immunol 2014 ; 32 : 513-45.

2. Meraz MA, White JM, Sheehan KC, et al. Targeted disruption of the Statl gene in mice reveals unexpected physiologic specificity in the JAK-STAT signaling pathway. Cell $1996 ; 84: 431-42$

3. Fink K, Grandvaux N. STAT2 and IRF9: Beyond ISGF3. JAKSTAT 2013 ; 2 : e27521.

4. Blaszczyk K, Olejnik A, Nowicka $H$, et al. STAT2 IRF9 directs a prolonged ISGF3-like transcriptional response and antiviral activity in the absence of STATl. Biochem J 2015 ; 466 : 511-24.

5. Johnson ES. Protein modification by SUMO. Annu Rev Biochem $2004 ; 73$ : 355-82

6. Lamoliatte F, Caron D, Durette C, et al. Large-scale analysis of lysine SUMOylation by SUMO remnant immunoaffinity profiling. Nat Commun $2014 ; 5$ : 5409.

7. Begitt A, Droescher M, Knobeloch KP, Vinkemeier U. SUMO conjugation of STATl protects cells from hyperresponsiveness to IFNgamma. Blood 2011; 118 : 1002-7.

8. Maarifi G, Maroui MA, Dutrieux J, et al. Small ubiquitin-like modifier alters IFN response. J Immunol $2015 ; 195$ : 2312-24.

9. Chelbi-Alix MK, Quignon F, Pelicano L, et al. Resistance to virus infection conferred by the interferon-induced promyelocytic leukemia protein. J Virol 1998 ; 72 : 1043-51.

10. Geoffroy MC, Chelbi-Alix MK. Role of promyelocytic leukemia protein in host antiviral defense. J Interferon Cytokine Res $2011 ; 31: 145-58$.

11. Regad T, Chelbi-Alix MK. PML, un nouvel intermédiaire de l'effet antiviral de l'interféron. Med Sci (Paris) $2002 ; 18: 25-7$.

12. Maroui MA, દl Asmi F, Dutrieux J, et al. Implication des corps nucléaires PML dans l'immunité intrinsèque et innée. Med Sci (Paris) 2014 ; $30: 765-71$

13. Tatham MH, Geoffroy MC, Shen L, et al. RNF4 is a poly-SUMO-specific $\varepsilon 3$ ubiquitin ligase required for arsenic-induced PML degradation. Nat Cell Biol 2008 ; $10: 538-46$.

14. Maroui MA, Kheddache-Atmane $S$, $\varepsilon \mid$ Asmi F, et al. Requirement of PML SUMO interacting motif for RNF4 or arsenic trioxide-induced degradation of nuclear PML isoforms. PLoS One $2012 ; 7$ : e44949.

15. عl Bougrini J, Dianoux L, Chelbi-Alix MK. PML positively regulates interferon gamma signaling. Biochimie $2011 ; 93: 389-98$.

16. Maroui MA, Pampin M, Chelbi-Alix MK. Promyelocytic leukemia isoform IV confers resistance to encephalomyocarditis virus via the sequestration of 3D polymerase in nuclear bodies. J Virol $2011 ; 85$ : 13164-73. 